\title{
What Comes After Immuno-Oncology Therapy for Kidney Cancer?
}

\author{
Haris Zahoor ${ }^{\mathrm{a}, *}$, Vinay Duddalwar ${ }^{\mathrm{a}}$, Anishka D’Souza ${ }^{\mathrm{a}}$, Axel S. Merseburger ${ }^{\mathrm{b}}$ and David I. Quinn ${ }^{\mathrm{a}}$ \\ ${ }^{a}$ Keck School of Medicine, University of Southern California, Norris Comprehensive Cancer Center, \\ Los Angeles, CA, USA \\ ${ }^{\mathrm{b}}$ Department of Urology, Campus Lübeck, University Hospital Schleswig-Holstein, Lübeck, Germany
}

\begin{abstract}
The treatment landscape of advanced renal cell carcinoma (RCC) is rapidly evolving. Immune checkpoint inhibitors (ICI) have now become the preferred first line treatment with the approval of nivolumab and ipilimumab combination in intermediate to poor risk patients. Combination/s of ICI with vascular endothelial growth factor (VEGF) inhibitors will also be approved in future. The optimal treatment of patients who progress on ICI-based therapies is not well defined as of yet. In this review, we discuss the data regarding various treatment options available in this space, their limitations, and also provide our opinion regarding treatment selection.
\end{abstract}

Keywords: Renal cell carcinoma, immuno-oncology, immune checkpoint inhibitor, tyrosine kinase inhibitors

\section{INTRODUCTION}

Renal cell carcinoma (RCC) accounts for approximately $3 \%$ of cancers in adults and is the most common kidney cancer accounting for $90-95 \%$ of cases [1]. In the US, an estimated 73,820 new cases with an estimated 14,770 deaths will occur from RCC in 2019 [2].

Surgical resection is the treatment of choice for patients with localized RCC, and there is no adjuvant treatment that has shown an improvement in overall survival (OS) after surgical resection [3]. Approximately $25-30 \%$ of patients present with metastatic disease and $30 \%$ of patients who are treated for localized RCC develop recurrent disease [4, 5]. These patients with metastatic, advanced, or recurrent RCC are then treated with systemic therapy, which

\footnotetext{
${ }^{*}$ Correspondence to: Haris Zahoor, MD, MS, Keck School of Medicine, University of Southern California, Norris Comprehensive Cancer Center, 1441 Eastlake Ave. NOR 3447, Los Angeles, CA 90035, USA. Tel.: +1 323865 3901; Fax: +1 323865 0061; E-mail: haris.zahoor@med.usc.edu.
}

dramatically changed with the introduction of targeted agents like vascular endothelial growth factor (VEGF) inhibitors (such as bevacizumab, sunitinib, pazopanib, axitinib and cabozantinib) or mammalian target of rapamycin (mTOR) inhibitors like temsirolimus and everolimus [6]. Although these agents have significantly improved outcomes, they rarely result in complete responses, and resistance to these agents is inevitable, leading to progressive disease (PD) in virtually all patients $[7,8]$.

Renal cell carcinoma is an immune-responsive tumor and high dose IL-2 has been used in select patients leading to complete and durable responses in a subset of them [9]. Improved understanding of $\mathrm{T}$ cell function and associated immunosuppressive molecules such as cytotoxic $\mathrm{T}$ lymphocyte-associated protein 4 (CTLA-4), program death 1 (PD-1) and PD-1 ligand 1 (PD-L1), called immune checkpoints, have led to the development of novel immunotherapies [10]. Nivolumab, an immune checkpoint inhibitor (ICI) in the form of a monoclonal antibody directed at PD-1, has been investigated for 
the treatment of refractory metastatic RCC. In a randomized phase III trial, Checkmate 025, nivolumab was shown to improve overall survival (OS) as compared to everolimus, a mammalian target of rapamycin (mTOR) inhibitor (Hazard Ratio (HR), $0.73 ; p=0.002)$. The overall response rate (ORR) with nivolumab was $25 \%$ vs. $5 \%$ with everolimus $(p<0.001)$, although complete response was rare $(1 \%)$. The treatment was well tolerated. Based on these data, nivolumab was approved by FDA as a first ICI in RCC treatment and became a preferred second line treatment option [11].

ICI either alone, or more often in combination with another ICI or a VEGF inhibitor, have now been introduced in the first line setting. Here we discuss data regarding these agents.

\section{NIVOLUMAB AND IPILIMUMAB COMBINATION}

Combination treatment with nivolumab and ipilimumab, an anti-CTLA 4 antibody, was investigated in untreated RCC patients in a randomized phase III trial, Checkmate 214 [12]. As compared to sunitinib, the nivolumab and ipilimumab combination led to improved OS $(\mathrm{HR}, 0.63 ; P<0.001)$ in intermediate or poor-risk patients. The median progression-free survival PFS was 11.6 months and 8.4 months, respectively (HR, $0.82 ; p=0.03$, not significant per the prespecified 0.009 threshold). The ORR was $42 \%$ vs. $27 \%(P<0.001)$, and the complete remission $(\mathrm{CR})$ rate was $9 \%$ vs. $1 \%$ in nivolumab and ipilimumab arm as compared to sunitinib. Based on these data, this combination was approved by FDA for intermediate or poor-risk RCC patients [13,14]. The situation is more complex in good-risk patients where response and PFS favor sunitinib over nivolumab and ipilimumab, but long term follow-up for overall survival data is required.

\section{AXITINIB AND PEMBROLIZUMAB COMBINATION}

A randomized phase III trial evaluated the combination of axitinib and pembrolizumab in untreated advanced or metastatic RCC compared to sunitinib. The co-primary endpoints of this trial were OS and PFS in the intention to treat population. The trial met both of its primary endpoints and showed that treatment with the combination of axitinib and pembrolizumab, lowers the risk of death (HR for death, $0.53 ; 95 \%$ CI, $0.38-0.74 ; P<0.0001)$ and disease progression (HR for disease progression or death, $0.69 ; 95 \% \mathrm{CI}, 0.57-0.84 ; P<0.001)$. The ORR was $59.3 \%$ (95\% CI, 54.5-63.9) in the combination group and $35.7 \%$ (95\% CI, 31.1 to 40.4 ) in the sunitinib group $(P<0.001)$. These favorable outcomes with combination treatment were observed across all risk groups and regardless of PD-L1 expression [15].

\section{AXITINIB AND AVELUMAB COMBINATION}

JAVELIN Renal 101 investigated the combination of axitinib and avelumab in untreated RCC patients with metastatic or advanced disease. The co-primary endpoints of this randomized phase III trial were PFS and OS in PD-L1+ patients. The trial met one of its co-primary endpoints with an improvement in PFS. Median PFS was 13.8 versus 7.2 months in the combination arm compared to the sunitinib arm (HR, 0.61; $p<0.001)$ in PD-L1+ patients. OS data was immature at the time of presentation. The median PFS in the overall population, a key secondary endpoint, was 13.8 versus 8.4 months in the combination arm compared to the sunitinib arm (HR, $0.69 ; p<0.001)$. The ORR and CR rate in PD-L1+ patients were 55\% and $4 \%$ in the combination arm as compared to $26 \%$ and $2 \%$ in the sunitinib arm [16].

\section{BEVACIZUMAB AND ATEZOLIZUMAB COMBINATION}

Similarly, a randomized phase III trial met one of its co-primary endpoint demonstrating superiority of combination of atezolizumab, an anti-PD-L1 antibody, with bevacizumab, as compared to sunitinib monotherapy [17]. The co-primary endpoints of the trial were PFS by investigator-assessment in PD-L1+ patients and OS in intention to treat population (IIT). The PFS in the PD-L1+ patients was 11.2 months $(8.9,15.0)$ in the combination arm vs. 7.7 months (6.8-9.7) in the sunitinib arm (HR, 0.74; 95\% CI: $0.57-0.96 ; p=0.02)$. The OS in the IIT population was immature at the time of data presentation. The ORR in the PD-L1+ patients was 43\% (35-50) in the combination arm as compared to $35 \%(28-42)$ in the sunitinib arm. The CR rate in the PD-L1+ patients was $9 \%$ in the combination arm as compared to $4 \%$ in the sunitinib arm. 


\section{PEMBROLIZUMAB MONOTHERAPY}

Similarly, pembrolizumab monotherapy for treatment naïve patients has also shown promising clinical activity in a phase II trial [18]. The ORR was $38.2 \%(29.1-47.9)$ and CR $2.7 \%$ in all treated patients. Patients with positive PD-L1 expression had an ORR of $50.0 \%(34.9-65.1)$ as compared with 26.4\% (15.3-40.3) seen in PD-L1 negative patients as defined by the investigator assessment. The median PFS was $8.7(6.7-12.2)$ and median OS was not reached.

\section{CABOZANTINIB}

Based on Meteor trial data as described later in this article, cabozantinib was investigated in intermediate and poor-risk previously untreated RCC in the randomized phase II CABOSUN trial [19]. The PFS with cabozantinib was 8.6 months $(95 \% \mathrm{CI}$ : $6.8,14.0)$ compared with 5.3 months $(95 \%$ CI: 3.0, 8.2 ) for patients taking sunitinib (HR $0.48 ; 95 \% \mathrm{CI}$ : $0.31,0.74 ; p=0.0008$ ) [20]. The FDA extended the approval for cabozantinib to include first line treatment naïve RCC patient on December 19, 2017, based on these results. This trial tried to answer an important question regarding the added clinical benefit of MET and AXL inhibition in addition to VEGF pathway inhibition with cabozantinib. However, several limitations of the trial have been highlighted [21] including an underperformance of sunitinib arm (5.6 months) as compared to historical data (10.6 months) from the registration trial [22] and more recent data (8.4 months) from the Checkmate 214 trial [12]. Based on a composite assessment of these datasets, an ICI based therapy has been adopted as a standard of care (SOC) for untreated RCC patients with the option of cabozantinib usually reserved for patients who are not candidates for an immune based therapy.

\section{WHAT COMES AFTER IO}

As discussed above, the first line treatment landscape of RCC is rapidly evolving. These changes in the treatment paradigm have raised several important clinical questions regarding the sequencing of treatment after patients progress on these immune based therapies. Although these patients will likely go on to receive TKIs (or other targeted treatments like mTOR inhibitors), the data regarding their efficacy and safety in this setting is needed. The main objective of this review is to summarize and discuss available data regarding various treatment options in these patients, our opinion regarding treatment sequence and selection, and future directions in this space.

Few retrospective analyses have evaluated the efficacy of targeted treatment in patients who have disease progression on ICI-based therapy [23-25]. The ORR ranged from $13-29 \%$ and PFS or Time to Treatment Failure (TTF) ranged from 6.4-6.6 months. These studies represent a heterogeneous patient population with several limitations but generally suggest feasibility and at least reasonable efficacy of targeted agents in this setting.

Another retrospective study evaluated the clinical outcomes of patients receiving second-line TKIs after progression on nivolumab and ipilimumab treatment as part of the Checkmate 214 study, thereby providing data in a more homogenous patient population [26]. In this analysis, sunitinib (51\%) was the most commonly used TKI followed by axitinib (24\%), pazopanib (18\%), and cabozantinib (6\%). The objective response rate was $36 \%$ and the disease control rate was $76 \%$. The median PFS for second-line TKI therapy was 8 months (95\% CI: 5-13). This study demonstrates clinical efficacy of TKIs after progression on front-line nivolumab and ipilimumab treatment.

Here we describe available data regarding the available agents in the treatment of refractory disease and specifically after PD on ICI-based therapy. (Table 1).

\section{AXITINIB}

Axitinib is a selective TKI against VEGF receptors 1,2 , and 3. In the randomized phase III AXIS trial it was compared against sorafenib in patients with metastatic RCC who had progressed on previous systemic therapy. Axitinib showed an improved PFS of 6.7 months vs. 4.7 months with sorafenib (HR, 0.665; $\mathrm{p}<0.0001)$. The ORR was $19 \%$ for patients receiving axitinib vs. $9 \%$ for sorafenib $(p=0 \cdot 0001)$ [27]. In patients who received cytokine-based immunotherapy as first line therapy, patient given axitinib on this study had a median PFS of 12.1 months vs. 6.5 months with sorafenib, suggesting efficacy for this agent after immunotherapy. Based on these data, axitinib was approved for patients with relapsed disease or refractory to cytokine and TKI therapy [13].

The activity of axitinib after progression on an ICI was evaluated in a prospective phase II trial where 
Table 1

Select TKIs after progression on an ICI

\begin{tabular}{|c|c|c|c|c|c|}
\hline Agent & $\begin{array}{l}\text { Number } \\
\text { (n) }\end{array}$ & Prior CPI & ORR & Median PFS & Median OS \\
\hline \multicolumn{6}{|l|}{ Axitinib } \\
\hline Ornstein et al. [28] & 38 & IO, IO-IO, IO-VEGF & $38.7 \%$ & 9.2 months & Not mature \\
\hline Barata et al. [24] & 14 & IO, IO-IO, IO-VEGF & $29 \%$ & 6.4 months & Not reported \\
\hline Albiges et al. [25] & 20 & IO monotherapy & $16 \%$ & $\begin{array}{l}\text { TTF } 10.0(0.2+, \\
19.3)\end{array}$ & $\begin{array}{r}\text { 1-year OS rate } \\
69 \%(34,88)\end{array}$ \\
\hline Auvray et al. [26] & 8 & IO-IO & Not reported & 7 months & Not reported \\
\hline \multicolumn{6}{|l|}{ Cabozantinib } \\
\hline Choueiri et al. [33] & 69 & $\mathrm{IO}, \mathrm{IO}+\mathrm{VEGF}$ & $33 \%$ & 6.6 months (TTF) & $\begin{array}{l}\text { 1-year OS rate } \\
53 \%\end{array}$ \\
\hline Barata et al. [24] & 3 & IO, IO-IO, IO-VEGF & $33 \%$ & Not reached & Not reported \\
\hline Tivozanib [46] & $\begin{array}{l}\text { Not } \\
\text { available }\end{array}$ & Not available & $18 \%$ & 5.6 months & Not mature \\
\hline \multicolumn{6}{|l|}{ Sunitinib } \\
\hline Barata et al. [24] & 4 & IO, IO-IO, IO-VEGF & $\begin{array}{l}\mathrm{SD} \text { in } 50 \% \text { and } \mathrm{PD} \text { in } \\
50 \%\end{array}$ & 2.9 months & Not reported \\
\hline Auvray et al. [26] & 17 & IO-IO & Not reported & 8 months & Not reported \\
\hline \multicolumn{6}{|l|}{ Pazopanib } \\
\hline Albiges et al. [25] & 14 & IO monotherapy & $\begin{array}{l}\mathrm{SD} \text { in } 64 \% \text { and } \mathrm{PD} \text { in } \\
36 \%\end{array}$ & TTF: 4.8 months & $\begin{array}{l}\text { 1-year OS rate } \\
61 \%\end{array}$ \\
\hline Barata et al. [24] & 7 & IO, IO-IO, IO-VEGF & $43 \%$ & 5.6 months & \\
\hline
\end{tabular}

axitinib was given on an individualized dosing algorithm. The primary endpoint of the trial was PFS. Thirty-eight patients were enrolled with a median age of 64 and good performance status of $>80 \%$ in $89 \%$. IMDC risk categories were $16 \%$ favorable, $66 \%$ intermediate and $19 \%$ poor risk. More than $70 \%$ patient had received $\geq 2$ prior therapies. Most recent therapy was anti PD-1 in $89 \%$ of the cases $(63 \%$ nivolumab monotherapy; $17 \%$ nivolumab and ipilimumab; $9 \%$ other) and anti PD-L1 in $11 \%$ of the cases $(6 \%$ atezolizumab, 3\% bevacizumab and atezolizumab; $3 \%$ durvalumab). The estimated median PFS in the evaluable patients was 9.2 months with $54 \%$ patients were still on the treatment at the time of presentation. The ORR was $38.7 \%$ whereas SD and PD was found in $48.3 \%$ and $12.9 \%$ respectively. There were no unexpected toxicities related to axitinib observed on the study [28].

The retrospective studies discussed above have also reported data on axitinib use in patients with progression on an ICI. In one study, 20/56 received axitinib after progression on an ICI with a median TTF of $10.0(0.2+, 19.3)$, one-year survival rate of 69 $\%(34,88)$, and ORR of $16 \%$ [25]. Similarly, a second study reported 14/28 patients who received axitinib after progression on an ICI with a median PFS of 6.4 months (4.7-8.1) and an ORR of $29 \%$ [24]. In patients progressing on nivolumab and ipilimumab, the median PFS with subsequent axitinib treatment was 7 months (95\% CI, 5-NR) [26].

\section{CABOZANTINIB}

Cabozantinib is an oral TKI which targets multiple kinases involved in RCC, including AXL, MET, and VEGF receptors [29]. It was approved for use in the refractory setting based on the phase III METEOR trial where cabozantinib showed an improved PFS as compared to everolimus (7.4 months vs. 3.8 months; HR, $0.58 ; 95 \% \mathrm{CI}, 0.45$ to $0.75 ; p<0.001)$. The response rates were $21 \%$ in cabozantinib arm vs. $5 \%$ with everolimus $(p<0.001)$ [30]. In the updated results, cabozantinib was found to have improved overall survival with median overall survival of 21.4 months (95\% CI 18.7-not estimable) vs. 16.5 months (14.7-18.8) with everolimus (HR, 0.66; 95\% CI, $0.53-0.83 ; p=0.0003$ ) [31]. In a subgroup analysis of patients with bone metastases, cabozantinib showed an improved PFS as compared to everolimus (7.4 months vs. 2.7 months; HR, 0.33; 95\% CI, 0.21-0.51). Similarly, OS, ORR and rate of skeletal events favored cabozantinib over everolimus. These data may suggest benefit of cabozantinib in patients with bone metastases. However, the PFS with cabozantinib in patients with bone metastases (7.4 months) was numerically similar to overall population (7.4 months), and therefore one could argue that these data infact suggest that everolimus has poor outcomes in patients with bone metastases [32].

A retrospective review of cabozantinib of 69 patients after PD on ICI was presented at ESMO 
2018. Median age on this study was 62 years (range 37-78) and median number of prior therapies was 2 (range 1-10). At the time of cabozantinib treatment, IMDC risk groups were $6 \%$ good, $67 \%$ intermediate and $27 \%$ poor. Most recent therapy was single agent ICI in 54\%, combination of ICI with a VEGF inhibitor in $35 \%$, and other therapies in $12 \%$. The ORR was $33 \%$ whereas SD and PD were found in $46 \%$ and $17 \%$ respectively. The median TTF was $6.6(95 \% \mathrm{CI}$ : 5.3-8.5) months and 35\% were still on treatment at the time of analysis. One-year OS rate was 53\% (95\% CI: $37 \%-66 \%$ ) [33].

In another retrospective study of VEGF TKIs after progression on ICI, 3/28 patients received cabozantinib after progression on an ICI with $33 \%$ ORR [24].

\section{TIVOZANIB}

This is a selective VEGFR 1, 2 and 3 inhibitor, which was evaluated in a phase III for untreated patients with metastatic RCC. Tivozanib showed an improvement in PFS as compared to sorafenib (11.9 vs. 9.1 months, $p=0.042$ ). The ORR for tivozanib, based on blinded independent radiology review, was $33.1 \%$ (95\% CI, 27.4-39.2) versus $23.3 \%$ (95\% CI, 18.3-29.0) for sorafenib. No improvement in OS was observed with tivozanib treatment. It should be noted that $61 \%$ patients in the sorafenib arm were crossed over to tivozanib arm due to PD whereas patients in the tivozanib arm were not allowed to cross over which might explain the lack of OS benefit [34].

Tivozanib compared to sorafenib was then investigated in refractory (failed at least two prior regimens) advanced or metastatic RCC. Tivozanib showed a $44 \%$ improvement in median PFS and $27 \%$ reduction in risk of progression or death (HR, 0.73; $p=0.02$ ). The median PFS was 5.6 months for tivozanib as compared to 3.9 months for sorafenib. The OS data was still immature whereas ORR was $18 \%$ with tivozanib compared to $8 \%$ for patients receiving sorafenib $(p=0.02)$. Of note, approximately $28 \%$ of patients in this trial received ICI in earlier lines of treatment and thus will be an important prospective dataset of a TKI use in the post ICI space [35].

\section{PAZOPANIB}

Pazopanib, an oral TKI against VEGF and PDGF receptors, was investigated in a phase III clinical trial in patients with advanced or metastatic RCC [36]. Patients who received pazopanib had improved PFS of 9.2 months vs. 4.2 months in patients who received placebo (HR, $0.46 ; p<0.0001)$. The ORR was $30 \%$ for patients in the pazopanib arm vs. $3 \%$ for placebo. It has been a commonly used initial agent in untreated metastatic RCC based on these data and favorable tolerability $[13,14]$.

No prospective data exists regarding the use of pazopanib in post ICI space, however an ongoing trial is accruing patients as discussed later in this article. In the retrospective review from Albiges et al., 14 patients received pazopanib with TTF of 4.8 (range, 0.6-11.1) months and 1-year OS survival rate of $61 \%$ (range, 27-84) [25]. Best response was SD in 9 patients whereas PD occurred in the remaining 5 patients. On the other hand, Barata et al. [24] reported 7 of 28 patients who received pazopanib after progression on an ICI with a median PFS of 5.6 months (1.2-10.0) and ORR of $43 \%$.

\section{SUNITINIB}

Sunitinib is an oral TKI against both VEGF and PDGF receptors [37]. It was evaluated in a phase III clinical trial which randomized patients with untreated metastatic RCC to sunitinib or IFN [22]. Patients who received sunitinib had an improved PFS (11 vs. 5 months; HR, $0.54 ; p<0.001$ ) and ORR ( $47 \%$ vs. $12 \%$; $p<0.001$ ) as compared to patients who received IFN. It has been used as a standard first line treatment options for metastatic renal cell carcinoma [13].

No prospective data regarding its use in the post ICI space exists. A retrospective study [24] has reported 4 patients treated with sunitinib with a median PFS of 2.9 (0.0-7.6). No responses were observed. SD was found in 50\% (2) and PD in 50\% (2) of patients. Similarly, in patients progressing on nivolumab and ipilimumab, the median PFS with subsequent sunitinib treatment was 8 months (95\% CI, 3-NR) [26].

\section{mTOR INHIBITORS}

Mammalian target of rapamycin is a serine/threonine kinase which acts as a downstream effector of the phosphoinositide 3-kinase/AKT pathway. mTOR inhibitors had been of great interest in RCC due to the association of mTOR pathway with $\mathrm{HIF}$, which is upregulated in majority cases of ccRCC due pVHL loss [38].

Everolimus was investigated in a phase III trial which compared everolimus with placebo for patients 
who had progressed on sunitinib or sorafenib [39]. Treatment with everolimus was associated with improved PFS (4.0 months vs 1.9 months; HR, $0.33 ; p<0.001)$ and an ORR of 1.8 percent. On the other hand, temsirolimus was investigated in a phase III trial where patients with poor- and some intermediate-risk RCC were randomized to temsirolimus, temsirolimus plus interferon (IFN) or IFN alone. Treatment with temsirolimus was associated with significant improvement in OS (10.9 versus 7.3 months; HR, 0.73; 95\% CI, 0.58-0.92). The median PFS (5.5 months vs. 3.1 months) and ORR (8.6\% vs. $4.8 \%$ ) were also improved with temsirolimus as compared to INF. No additional benefit from combining temsirolimus with IFN was observed [40]. Based on these data both everolimus and temsirolimus have been approved as potential systemic treatments in refractory and first line setting respectively [13]. However, clinically, their use has been pushed to highly refractory patients due to availability of multiple other active agents and the likelihood that major responders have alterations of the PI3K-Akt-mTOR pathway.

No prospective data regarding their use in the post ICI space exists. However, in the Albiges et al report [25], everolimus was used in 11 of 56 patients in post ICI period with a median TTF of 4.8 (range, 0.5-23.0) months and 1 year OS survival rate of 27 (range, 4-58).

\section{LENVATINIB PLUS EVEROLIMUS}

The combination of everolimus with a multi-kinase inhibitor, lenvatinib, has been investigated. In an open label randomized phase II trial, 153 patients with metastatic RCC with PD on a VEGF-targeted therapy were randomized to $1: 1: 1$ ratio to either lenvatinib, everolimus, or lenvatinib plus everolimus. The combination of lenvatinib plus everolimus led to improved PFS of 12.8 (95\% CI, 7.4-17.5) vs. 9.0 months (95\% CI, 5.6-10.2) in the lenvatinib arm, and 5.6 months (3.6-9.3) in the everolimus arm. PFS was statistically significantly superior with lenvatinib plus everolimus as compared to everolimus alone (HR, $0.45 ; p=0.003$ ) while there was no significant difference between lenvatinib alone and everolimus alone (HR, 0.62; $p=0.12$ ). The ORR with lenvatinib plus everolimus was $35 \%$ as compared to $39 \%$ with lenvatinib alone and $0 \%$ with everolimus alone $(p<0.0001$ for both comparisons) [41]. In a post-hoc updated analysis, the OS was also found to be significantly improved with lenvatinib plus everolimus as compared to everolimus alone (25.5 months [95\% CI 16.4-NE] vs. 15.4 months [11.8-19.6]; HR, 0.51; $95 \%$ CI, $0 \cdot 30-0 \cdot 88 ; p=0 \cdot 024)$. Based on these data, the combination of lenvatinib plus everolimus was approved by the FDA for the treatment of patients with metastatic refractory RCC [42].

Although no data regarding the efficacy of this combination in post-ICI space exists, it remains a reasonable option to consider.

\section{ONGOING TRIALS}

Several ongoing trials are investigating the role of TKIs, either alone or in combination with novel agents, for patients who have progressed on an ICI. Selected trials in this space are presented in Table 2.

\section{EXPERT OPINION}

The first line treatment landscape of RCC is rapidly evolving and ICI have become a new SOC. There are limited data regarding the outcomes of patients who progress on ICI [43]. Similarly, optimal management of patients who progress after ICI is not fully defined. However, it appears that VEGF TKIs have become a "de-facto" choice of treatment in the post immunotherapy space. VEGF inhibitors have immunomodulatory effects with a potential to enhance the anti-tumor activity of ICI, and this has been the basis of combination of these agents with ICI. However, it remains unclear if the combination will be superior to sequencing of these respective agents. Nonetheless, the preliminary antitumor activity of VEGF TKI after ICI, as discussed above, provides a basis to use these agents until more definitive data is forthcoming. A proposed treatment algorithm is presented in Fig. 1.

The preferred agent of choice also remains to be determined. Axitinib and tivozanib (full data awaited) have prospective data whereas cabozantinib has the largest retrospective data in this space. Cabozantinib also has in its favor the demonstration of OS improvement in a phase III trial over an active competitor (everolimus), whereas other agents have not produced this. Several factors including prior TKI used (if IO-VEGF combination is used), efficacy and safety will be considered in selection of a preferred TKI. The use of pazopanib and sunitinib in combination with ICI led to increased toxicity and thus limiting further development of either agent in these 
Table 2

Ongoing trials for patients who have progressed on ICI

\begin{tabular}{|c|c|c|}
\hline Agent or Approach & Brief Description & NCT Number \\
\hline Pazopanib [47] & $\begin{array}{l}\text { This is an international, multicenter, single arm phase II trial to } \\
\text { investigate the efficacy, safety and quality of life of pazopanib } \\
\text { treatment after previous therapy with immune checkpoint } \\
\text { treatment. This trial will provide first prospective evidence of } \\
\text { pazopanib use in the post ICI space. }\end{array}$ & NCT03200717 \\
\hline $\begin{array}{l}\text { Cabozantinib plus HIF } \\
\text { Inhibitor [48] }\end{array}$ & $\begin{array}{l}\text { Hypoxia inducible factor (HIF)-Inhibitor PT } 2977 \text { is being } \\
\text { evaluated in combination with cabozantinib in an open-label } \\
\text { phase } 2 \text { study in patients with advanced ccRCC. The cohort } 2 \text { of } \\
\text { this trial will enroll patients who have progressed on an ICI. }\end{array}$ & NCT03634540 \\
\hline $\begin{array}{l}\text { Cabozantinib plus } \\
\text { Glutaminase Inhibitor [49] }\end{array}$ & $\begin{array}{l}\text { This randomized, double-blind, placebo-controlled phase } 2 \text { study } \\
\text { will evaluate the combination of glutaminase inhibitor in } \\
\text { combination with cabozantinib in patients who progressed on } \\
\text { either one antiangiogenic therapy or nivolumab and ipilimumab. }\end{array}$ & NCT03428217 \\
\hline $\begin{array}{l}\text { Axitinib plus OX-40 } \\
\text { antibody [50] }\end{array}$ & $\begin{array}{l}\text { This is a phase II trial investigating combination of axitinib and } \\
\text { OX-40 antibody in patients who have progressed on ICI. }\end{array}$ & NCT03092856 \\
\hline Salvage ICI [51-53] & $\begin{array}{l}\text { Another area of clinical and research interest is treating patients } \\
\text { with another ICI as a salvage treatment at the time of PD. Several } \\
\text { ongoing trials are investigating the optimization and sequencing } \\
\text { of ICI in RCC and will provide data regarding this approach. }\end{array}$ & $\begin{array}{l}\text { NCT03203473 } \\
\text { NCT02917772 } \\
\text { NCT03117309 }\end{array}$ \\
\hline
\end{tabular}

combinations [44]. Since ICI are antibodies with long half-lives, caution should exercised regarding the sequence and appropriate washout time before starting these TKIs after PD on ICI, although in contradistinction the efficacy data for VEGF-IO combinations may suggest that overlapping exposure may improve efficacy even after progression on the ICI. The retrospective data have not shown an additional toxicity with VEGF TKI use in patients who progress on immunotherapy except for a potential early toxicity signals of ALT/AST elevation in one of the studies [24]. Three patients developed ALT/AST abnormalities ( 2 with Grade 3 and 1 with Grade 4 toxicity). Of these 3 patients, 2 started pazopanib 1 day after discontinuation of ICI (ipilimumab/nivolumab and atezolizumab/bevacizumab) while one patient started axitinib 4 weeks after nivolumab and ipilimumab discontinuation. The authors did not find any correlation between timing of TKI use and development of these toxicities but numbers are too small to make any definitive conclusion. Regardless of interaction with prior ICI, TKIs have long term chronic toxicities which can accumulate over time and significantly impair quality of life. Data regarding various optimization schemes of TKI use can be informative for clinicians and will evolve with our experience in the post ICI space [45].

Prior use of IO-IO vs. IO-VEGF combination may also impact the efficacy of subsequent treatment with a TKI. For example, in a retrospective

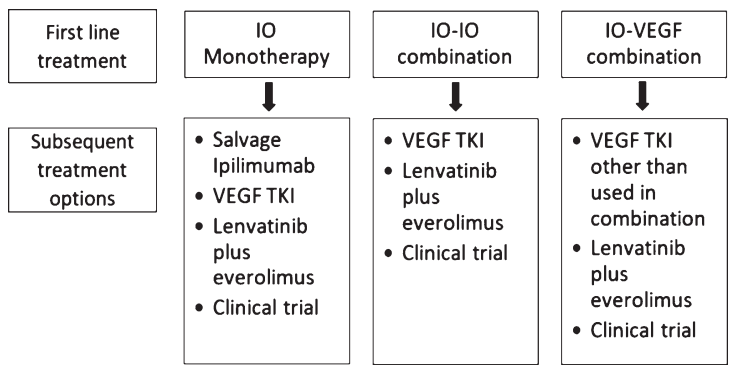

Fig. 1. Proposed systemic treatment algorithm after progression on ICI.

study, ORR was higher for patients previously treated with combination of two ICI compared with ICI plus anti-VEGF combination, although this was not statistically significant $(p=0.678)$ [24]. Similarly, another retrospective study also reported higher ORR ( $36 \%$ vs. $10 \%, p=0.039)$ for patients who were previously treated with ICI as compared to ICI plus anti-VEGF combination, although this didn't translate into improved PFS [23]. It should be noted that use of TKI after progression on a TKI has been a standard of care in RCC established by AXIS trial data [27].

In conclusion, prospective data regarding the efficacy, safety and appropriate treatment selection are needed in patients who have progressed on ICI. Similarly, novel combinations with VEGF TKI are being explored in this space. For now, VEGF TKIs will 
continue to be used in this setting based on their relative efficacy and toxicity profile.

\section{ACKNOWLEDGMENTS}

The authors have no acknowledgments.

\section{FUNDING}

The authors report no funding.

\section{CONFLICTS OF INTEREST}

HZ reports honoraria and advisory board activity for Exelixis.

VD reports being an advisor to Deeptek, and consultant to Intuitive systems.

$\mathrm{AD}$ reports no $\mathrm{COI}$.

ASM reports consultancy fees and/or was speaker from Amgen, Merck Sharp \& Dohme, Clovis, Bristol-Myers Squibb, Astellas, Pfizer, Sanofi, Roche, AstraZeneca.

DIQ reports honoraria and advisory board activity for Pfizer, Novartis, BMS, Exelixis, Bayer, Merck, EMD Serono.

\section{REFERENCES}

[1] Gupta K, Miller JD, Li JZ, Russell MW, Charbonneau C. Epidemiologic and socioeconomic burden of metastatic renal cell carcinoma (mRCC): A literature review. Cancer Treatment Reviews. 2008;34(3):193-205.

[2] Siegel RL, Miller KD, Jemal A. Cancer statistics, 2019. CA: A Cancer Journal for Clinicians. 2019;69(1):7-34.

[3] Rini BI, Campbell SC, Escudier B. Renal cell carcinoma. Lancet. 2009;373(9669):1119-32.

[4] Motzer RJ, Bander NH, Nanus DM. Renal-cell carcinoma. The New England Journal of Medicine. 1996;335(12): 865-75.

[5] Czarnecka AM, Kornakiewicz A, Kukwa W, Szczylik C. Frontiers in clinical and molecular diagnostics and staging of metastatic clear cell renal cell carcinoma. Future Oncology. 2014;10(6):1095-111.

[6] Zahoor H, Rini BI. Emerging growth factor receptor antagonists for the treatment of renal cell carcinoma. Expert Opinion on Emerging Drugs. 2016;21(4):431-40.

[7] Choueiri TK. VEGF inhibitors in metastatic renal cell carcinoma: Current therapies and future perspective. Current Clinical Pharmacology. 2011;6(3):164-8.

[8] Albiges L, Salem M, Rini B, Escudier B. Vascular endothelial growth factor-targeted therapies in advanced renal cell carcinoma. Hematology/Oncology Clinics of North America. 2011;25(4):813-33.

[9] Fyfe G, Fisher RI, Rosenberg SA, Sznol M, Parkinson DR, Louie AC. Results of treatment of 255 patients with metastatic renal cell carcinoma who received highdose recombinant interleukin-2 therapy. Journal of Clinical
Oncology: Official journal of the American Society of Clinical Oncology. 1995;13(3):688-96.

[10] Carlo MI, Voss MH, Motzer RJ. Checkpoint inhibitors and other novel immunotherapies for advanced renal cell carcinoma. Nature Reviews Urology. 2016;13(7):420-31.

[11] Motzer RJ, Escudier B, McDermott DF, George S, Hammers HJ, Srinivas S, et al. Nivolumab versus Everolimus in Advanced Renal-Cell Carcinoma. The New England Journal of Medicine. 2015;373(19):1803-13.

[12] Motzer RJ, Tannir NM, McDermott DF, Aren Frontera O, Melichar B, Choueiri TK, et al. Nivolumab plus Ipilimumab versus Sunitinib in Advanced Renal-Cell Carcinoma. The New England Journal of Medicine. 2018;378(14):1277-90.

[13] National Comprehensive Cancer Network. Clinical practice guidelines in oncology: Kidney Cancer. Available at: https://http://www.ncen.org/professionals/physician_gls/ pdf/kidney.pd. 2019.

[14] Powles T, Albiges L, Staehler M, Bensalah K, Dabestani S, Giles RH, et al. Updated European Association of Urology Guidelines Recommendations for the Treatment of Firstline Metastatic Clear Cell Renal Cancer. European urology. 2017.

[15] Rini BI, Plimack ER, Stus V, Gafanov R, Hawkins R, Nosov $\mathrm{D}$, et al. Pembrolizumab plus Axitinib versus Sunitinib for Advanced Renal-Cell Carcinoma. The New England Journal of Medicine. 2019;380(12):1116-27.

[16] Motzer RJ, Penkov K, Haanen J, Rini B, Albiges L, Campbell MT, et al. Avelumab plus Axitinib versus Sunitinib for Advanced Renal-Cell Carcinoma. The New England Journal of Medicine. 2019;380(12):1103-15.

[17] Robert J. Motzer TP, Michael B. Atkins, Bernard Escudier, David F. McDermott, Cristina Suarez, et al. IMmotion151: A Randomized Phase III Study of Atezolizumab Plus Bevacizumab vs Sunitinib in Untreated Metastatic Renal Cell Carcinoma (mRCC). DOI: 101200/JCO2018366_suppl578 Journal of Clinical Oncology 36, no 6_suppl (February 2018) 578-578

[18] David F. McDermott J-LL CS, Frede Donskov, Jahangeer Malik, Boris Yakovlevich Alekseev, James M. G. Larkin, et al. Pembrolizumab monotherapy as first-line therapy in advanced clear cell renal cell carcinoma (accRCC): Results from cohort A of KEYNOTE-427. J Clin Oncol. 2018;36(suppl; abstr 4500).

[19] Choueiri TK, Halabi S, Sanford BL, Hahn O, Michaelson MD, Walsh MK, et al. Cabozantinib Versus Sunitinib As Initial Targeted Therapy for Patients With Metastatic Renal Cell Carcinoma of Poor or Intermediate Risk: The Alliance A031203 CABOSUN Trial. Journal of Clinical Oncology: Official Journal of the American Society of Clinical Oncology. 2017;35(6):591-7.

[20] Choueiri TK, Hessel C, Halabi S, Sanford B, Michaelson MD, Hahn $\mathrm{O}$, et al. Cabozantinib versus sunitinib as initial therapy for metastatic renal cell carcinoma of intermediate or poor risk (Alliance A031203 CABOSUN randomised trial): Progression-free survival by independent review and overall survival update. European Journal of Cancer. 2018;94:115-25.

[21] Rini BI, Vogelzang NJ. Future Challenges for Drug Development in Renal Cell Carcinoma. Journal of Clinical Oncology: Official Journal of the American Society of Clinical Oncology. 2017;35(6):577-9.

[22] Motzer RJ, Hutson TE, Tomczak P, Michaelson MD, Bukowski RM, Rixe O, et al. Sunitinib versus interferon alfa in metastatic renal-cell carcinoma. The New England Journal of Medicine. 2007;356(2):115-24. 
[23] Nadal R, Amin A, Geynisman DM, Voss MH, Weinstock M, Doyle J, et al. Safety and clinical activity of vascular endothelial growth factor receptor (VEGFR)tyrosine kinase inhibitors after programmed cell death 1 inhibitor treatment in patients with metastatic clear cell renal cell carcinoma. Annals of Oncology: Official Journal of the European Society for Medical Oncology / ESMO. 2016;27(7):1304-11.

[24] Barata PC, De Liano AG, Mendiratta P, Crolley V, Szabados B, Morrison L, et al. The efficacy of VEGFR TKI therapy after progression on immune combination therapy in metastatic renal cell carcinoma. British Journal of Cancer. 2018;119(2):160-3.

[25] Albiges L, Fay AP, Xie W, Krajewski K, McDermott DF, Heng DY, et al. Efficacy of targeted therapies after PD-1/PDL1 blockade in metastatic renal cell carcinoma. European Journal of Cancer. 2015;51(17):2580-6.

[26] Auvray M, Auclin E, Barthelemy P, Bono P, KellokumpuLehtinen P, Gross-Goupil M, et al. Second-line targeted therapies after nivolumab-ipilimumab failure in metastatic renal cell carcinoma. European Journal of Cancer. 2019;108:33-40.

[27] Rini BI, Escudier B, Tomczak P, Kaprin A, Szczylik C, Hutson TE, et al. Comparative effectiveness of axitinib versus sorafenib in advanced renal cell carcinoma (AXIS): A randomised phase 3 trial. Lancet. 2011;378(9807): 1931-9.

[28] Moshe Chaim Ornstein SKP, Laura S. Wood, Kimberly D Allman, Allison Martin, Jorge A. Garcia, et al. Prospective phase II multi-center study of individualized axitinib (Axi) titration for metastatic renal cell carcinoma (mRCC) after treatment with PD-1 / PD-L1 inhibitors. DOI: 101200/JCO20183615_supp14517 Journal of Clinical Oncology 36, no 15_suppl (May 2018) 4517-4517.

[29] Yakes FM, Chen J, Tan J, Yamaguchi K, Shi Y, Yu P, et al. Cabozantinib (XL184), a novel MET and VEGFR2 inhibitor, simultaneously suppresses metastasis, angiogenesis, and tumor growth. Molecular Cancer Therapeutics. 2011;10(12):2298-308.

[30] Choueiri TK, Escudier B, Powles T, Mainwaring PN, Rini BI, Donskov F, et al. Cabozantinib versus Everolimus in Advanced Renal-Cell Carcinoma. The New England Journal of Medicine. 2015;373(19):1814-23.

[31] Choueiri TK, Escudier B, Powles T, Tannir NM, Mainwaring PN, Rini BI, et al. Cabozantinib versus everolimus in advanced renal cell carcinoma (METEOR): Final results from a randomised, open-label, phase 3 trial. The Lancet Oncology. 2016;17(7):917-27.

[32] Escudier B, Powles T, Motzer RJ, Olencki T, Aren Frontera O, Oudard S, et al. Cabozantinib, a New Standard of Care for Patients With Advanced Renal Cell Carcinoma and Bone Metastases? Subgroup Analysis of the METEOR Trial. Journal of Clinical Oncology: Official Journal of the American Society of Clinical Oncology. 2018;36(8):76572.

[33] B.A. McGregor AL, W. Xie, J.A. Steinharter, D.J. Martini, P.V. Nuzzo, et al. Activity of cabozantinib (cabo) after PD-1/PD-L1 immune checkpoint blockade (ICB) in metastatic clear cell renal cell carcinoma (mccRCC). Annals of Oncology (2018) 29 (suppl_8): viii303-viii331 101093/annonc/mdy283.

[34] Motzer RJ, Nosov D, Eisen T, Bondarenko I, Lesovoy $\mathrm{V}$, Lipatov $\mathrm{O}$, et al. Tivozanib versus sorafenib as initial targeted therapy for patients with metastatic renal cell carcinoma: Results from a phase III trial. Journal of Clinical
Oncology: Official journal of the American Society of Clinical Oncology. 2013;31(30):3791-9.

[35] Brian I. Rini SKP, Bernard Escudier, Michael B. Atkins, Thomas E. Hutson, Camillo Porta, et al. TIVO-3: A phase III, randomized, controlled, multicenter, open-label study to compare tivozanib to sorafenib in subjects with refractory advanced renal cell carcinoma (RCC). J Clin Oncol. 2019;37(supp1 7S; abstr 541).

[36] Sternberg CN, Davis ID, Mardiak J, Szczylik C, Lee E, Wagstaff J, et al. Pazopanib in locally advanced or metastatic renal cell carcinoma: Results of a randomized phase III trial. Journal of Clinical Oncology: Official Journal of the American Society of Clinical Oncology. 2010;28(6):1061-8.

[37] Mendel DB, Laird AD, Xin X, Louie SG, Christensen JG, Li G, et al. In vivo antitumor activity of SU11248, a novel tyrosine kinase inhibitor targeting vascular endothelial growth factor and platelet-derived growth factor receptors: Determination of a pharmacokinetic/pharmacodynamic relationship. Clinical Cancer Research: An Official Journal of the American Association for Cancer Research. 2003;9(1):327-37.

[38] Battelli C, Cho DC. mTOR inhibitors in renal cell carcinoma. Therapy. 2011;8(4):359-67.

[39] Motzer RJ, Escudier B, Oudard S, Hutson TE, Porta C, Bracarda $\mathrm{S}$, et al. Efficacy of everolimus in advanced renal cell carcinoma: A double-blind, randomised, placebo-controlled phase III trial. Lancet. 2008;372(9637):449-56.

[40] Hudes G, Carducci M, Tomczak P, Dutcher J, Figlin R, Kapoor A, et al. Temsirolimus, interferon alfa, or both for advanced renal-cell carcinoma. The New England Journal of Medicine. 2007;356(22):2271-81.

[41] Motzer RJ, Hutson TE, Ren M, Dutcus C, Larkin J. Independent assessment of lenvatinib plus everolimus in patients with metastatic renal cell carcinoma. The Lancet Oncology. 2016;17(1):e4-5.

[42] U.S. Food and Drug Administration. Approval of Lenvatinib in combination with Everolimus. 2016. Available at http://www.fda.gov/Drugs/InformationOnDrugs/Approved Drugs/ucm501070.htm [ Last accessed on 15 August 2016].

[43] Zahoor H, Barata PC, Jia X, Martin A, Allman KD, Wood LS, et al. Patterns, predictors and subsequent outcomes of disease progression in metastatic renal cell carcinoma patients treated with nivolumab. Journal for Immunotherapy of Cancer. 2018;6(1):107.

[44] Amin A, Plimack ER, Ernstoff MS, Lewis LD, Bauer TM, McDermott DF, et al. Safety and efficacy of nivolumab in combination with sunitinib or pazopanib in advanced or metastatic renal cell carcinoma: The CheckMate 016 study. Journal for Immunotherapy of Cancer. 2018;6(1):109.

[45] Zahoor H, Rini BI, Ornstein MC. Extended therapy breaks from VEGFR TKI therapy in renal cell carcinoma: Sometimes less is more. Oncotarget. 2018;9(18):14036-7.

[46] https://investor.aveooncology.com/news-releases/newsrelease-details/aveo-oncology-announces-phase-3-tivo-3trial-tivozanib-renal. 2018.

[47] Study of Efficacy, Safety, and Quality of Life of Pazopanib in Patients With Advanced and/or Metastatic Renal Cell Carcinoma After Prior Checkpoint Inhibitor Treatment (IO-PAZ). https://clinicaltrialsgov/ct2/show/NCT03200717?term=NC T03200717\&rank=1.

[48] A Trial of PT2977 in Combination With Cabozantinib in Patients With Clear Cell Renal Cell Carcinoma (ccRCC). https://clinicaltrialsgov/ct2/show/NCT03634540?term=NC T03634540\&rank $=1$. 
[49] CANTATA: CB-839 With Cabozantinib vs. Cabozantinib With Placebo in Patients With Metastatic Renal Cell Carcinoma (CANTATA). https://clinicaltrialsgov/ct2/show/ NCT03428217?term=NCT03428217\&rank $=1$.

[50] Axitinib With or Without Anti-OX40 Antibody PF04518600 in Treating Patients With Metastatic Kidney Cancer. https://clinicaltrialsgov/ct2/show/NCT03092856.

[51] Study of Optimized Management of Nivolumab Based on Response in Patients With Advanced RCC (OMNIVORE Study). https://clinicaltrialsgov/ct2/show/NCT03203473.
[52] Tailored ImmunoTherapy Approach With Nivolumab in Subjects With Metastatic or Advanced Renal Cell Carcinoma (TITAN-RCC). https://clinicaltrialsgov/ct2/show/NC T02917772.

[53] Study of Front Line Therapy With Nivolumab and Salvage Nivolumab + Ipilimumab in Patients With Advanced Renal Cell Carcinoma. https://wwwclinicaltrialsgov/ct2/ show/NCT03117309. 